\title{
Characterization of intense pressure pulse through cylindrical hole
}

\author{
Koji Aizawa ${ }^{1,2, *}$ and Takumi Kobayashi ${ }^{1}$ \\ ${ }^{1}$ Department of Electrical and Electronics Engineering, Graduate School of Engineering, Kanazawa Institute of Technology, \\ Ohgigaoka 7-1, Nonoichi, 921-8501 Japan \\ ${ }^{2}$ Center for Electric, Optic and Energy Applications, Kanazawa Institute of Technology, \\ Ohgigaoka 7-1, Nonoichi, 921-8501 Japan
}

(Received 2 December 2019, Accepted for publication 10 January 2020)

Keywords: Pressure pulse, Cylindrical through-hole, Underwater spark discharge, Simulation PACS number: 43.20Mv [doi:10.1250/ast.41.776]

\section{Introduction}

Intense compressive pressure pulses with short duration, which are known as shockwave, are used for widely used for noninvasive treatment such as calculi destruction in human body [1]. On the other hand, Ohl and co-workers have been reported the cell detachment and molecular uptake into cells by a single focused shock wave generated using an extracorporeal shock wave lithotripter [2]. Hasebe and co-workers also have reported the molecular uptake into the human cells seeded on the culture dish bottom without focusing single underwater shockwave [3]. A single pressure pulse is sufficient to permeabilize the stratum corneum (SC) as well as the cell membrane. Therefore, this becomes one of promising candidates for transdermal drug delivery [4].

To realize the targeted transdermal drug delivery, it is necessary to irradiate a specific area range with a pressure pulse while keeping the sufficient intensity. Recently, we have reported the effect of a cylindrical hole on the propagation of the single underwater shock wave generated by the spark discharge [5]. The pressure pulse propagated through a cylindrical through-hole involved large pressure in comparison with that propagated in free space. However, the propagation characteristics of the pressure pulse through a cylindrical hole with several diameters are not investigated in detail. In this study, we numerically and experimentally investigated the effect of the cylindrical through-hole with different diameters on the propagation of single pressure pulse generated by underwater spark discharge.

\section{Preparation}

2.1. Pressure pulse generation and electrical measurement A high voltage is applied between two needle electrodes installed in water using an impulse voltage generator to induce a spark discharge and generate an underwater shockwave. This generator includes a charging circuit and a gap switch (GS). The boosted and rectified AC voltage is charged in five parallel-connected capacitors (each capacitance of $1,700 \mathrm{pF}$ ). When a GS turns on, the charged voltage in the capacitor is applied to the Tungsten needle electrode (tip radius is $25 \mu \mathrm{m}$ ) installed into a groove of Teflon block [3]. In this experiment, the GS interval and needle tip distance

\footnotetext{
*e-mail: aizawa@neptune.kanazawa-it.ac.jp
}

were fixed at $3 \mathrm{~mm}$ and $2 \mathrm{~mm}$, respectively. The applied voltage and the discharge current were measured using a high-voltage probe (Nissin Pulse Electronics, EP-50K) and a current transformer (Peason Electronics, 110). These waveforms were measured using a digital oscilloscope (Iwatsu, DS-5654A). Table 1 shows the summary of the charge and discharge parameters used in the pressure pulse generation.

2.2. Preparation of cylindrical through-hole and pressure measurement

Figure 1 shows the illustration of a cylindrical hole formed in the solid plate and of Teflon block with the discharge needles installed in a groove. The width and depth of a groove are respective $4 \mathrm{~mm}$. A polycarbonate (PC) was used as plate material because of faster sound speed than water at the same temperature, excellent electrical insulation and shock resistance. The diameter $\mathrm{D}$ of the cylindrical through-hole was varied from 0 to $5 \mathrm{~mm}$. The PC plate thickness, which corresponds to the hole length, was fixed at $5 \mathrm{~mm}$. This length is ten times larger than that the pulse width (multiply velocity by pulse duration). A through-hole center is located at the middle between the discharge needle tips. The PC plate with a through-hole was fixed on the Teflon brock with discharge electrodes. In this case, the entrance of a through-hole was located above $2 \mathrm{~mm}$ from the electrode. They were immersed in acrylic tank $(100 \times 200 \times 100 \mathrm{~mm})$ containing pure water. A part of the pressure pulse generated by spark discharge propagates through the water-filled cylindrical hole. The tip of a hydrophone sensor (Muller Platte Needle Probe, rise time $50 \mathrm{~ns}$, sensitive diameter $<0.5 \mathrm{~mm}$ ) was placed at the position of $1 \mathrm{~mm}$ away from the exit of a through-hole. In this experiment, the distance from the needle electrode to the tip of the hydrophone sensor was fixed at $8 \mathrm{~mm}$.

\subsection{Numerical simulation method}

In order to comprehend the pressure pulse propagation in a through-hole with different diameters, the numerical analysis was performed by finite-difference time-domain (FDTD) method using a cross-sectional two-dimensional (2D) model with a pressure source located on the central axis [6-8]. It is known that three-dimensional analysis is necessary. We however consider that this $2 \mathrm{D}$ model is useful to understand the pressure pulse propagation in the through-hole, because this through-hole is axially symmetric. The parameters using 
Table 1 Parameters of underwater spark discharge.

\begin{tabular}{ll}
\hline Parameters & average \pm S.D. \\
\hline Charged voltage & $10.6 \pm 0.4 \mathrm{kV}$ \\
Discharge current & $326 \pm 21 \mathrm{~A}$ \\
Discharge power & $1.78 \pm 0.11 \mathrm{MW}$ \\
Discharge Energy & $0.46 \pm 0.03 \mathrm{~J}$ \\
\hline
\end{tabular}

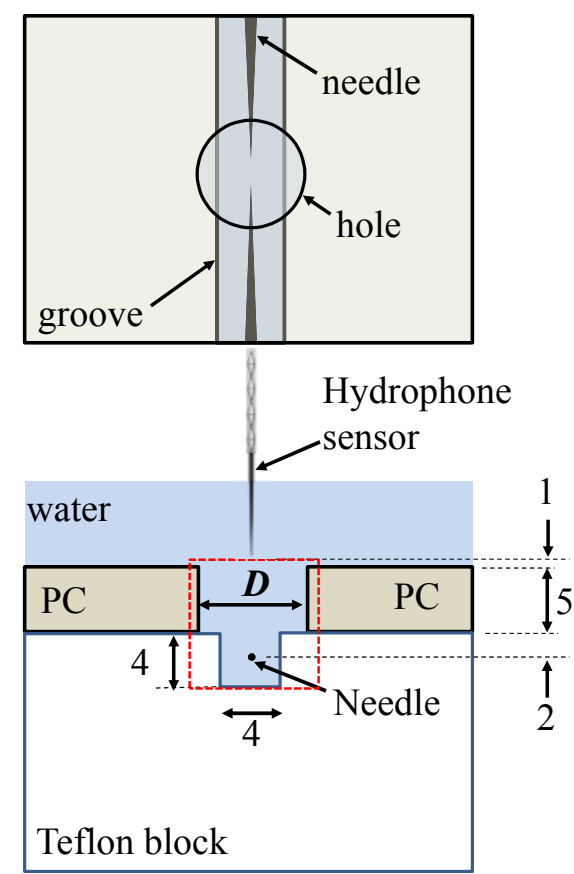

Fig. 1 Top and cross-sectional views of a cylindrical through-hole formed in the PC plate which was placed upward the discharge needles installed in a groove of the Teflon block. They are immersed in pure water. Simulation space $(10 \mathrm{~mm} \times 10 \mathrm{~mm})$ is shown as an area surrounded by red-dashed line.

Table 2 Parameters using 2D-FDTD simulation.

\begin{tabular}{ll}
\hline Parameters & Values \\
\hline Number of division & $400 \times 400$ \\
Increments in space, $\Delta x=\Delta y$ & $25 \mu \mathrm{m}$ \\
Increment in time, $\Delta t$ & $4.27 \mathrm{~ns}$ \\
Courant number & 0.256 in water \\
Input waveform & Gaussian \\
Formula of input temporal waveform & $200 e^{-\left(\frac{t}{t_{\mathrm{d}}}-5\right)^{2}}$ \\
Width of input waveform, $t_{\mathrm{d}}$ & $0.245 \mu \mathrm{s}$ \\
Boundary condition & Higdon's 2 nd-order \\
& absorption boundary \\
Nonlinear parameter of water & 3.5 \\
Density and sound velocity of water & $1,000 \mathrm{~kg} / \mathrm{m}^{3}$ and $1,500 \mathrm{~m} / \mathrm{s}$ \\
Density and sound velocity of PC & $1,198 \mathrm{~kg} / \mathrm{m}^{3}$ and $2,280 \mathrm{~m} / \mathrm{s}$ \\
Density and sound velocity of Teflon & $2,180 \mathrm{~kg} / \mathrm{m}^{3}$ and $1,380 \mathrm{~m} / \mathrm{s}$ \\
\hline
\end{tabular}

2D-FDTD was shown in Table 2. To calculate the propagation in the space $(10 \mathrm{~mm} \times 10 \mathrm{~mm}$, Fig. 1) including a PC plate with a through-hole and Teflon block, the increments in space $\Delta x=\Delta y=25 \mu \mathrm{m}$ and in time $\Delta t=4.27 \mathrm{~ns}$ were chosen. A Gaussian pulse with amplitude of $200 \mathrm{MPa}$ was applied to a point source as input signal. The propagation loss was neglect. Higdon's absorbing boundary condition was applied to the outer boundary of the analytical space [9]. The density and longitudinal velocity values of water $(1,000$ $\left.\mathrm{kg} / \mathrm{m}^{3}, 1,500 \mathrm{~m} / \mathrm{s}\right), \mathrm{PC}\left(1,198 \mathrm{~kg} / \mathrm{m}^{3}, 2,280 \mathrm{~m} / \mathrm{s}\right)$, and Teflon $\left(2,180 \mathrm{~kg} / \mathrm{m}^{3}, 1,380 \mathrm{~m} / \mathrm{s}\right)$ were used in this calculation. In order to discuss the first arrival wave in a liquid medium, transverse waves in solid media were not calculated.

\section{Results and discussion}

\subsection{Pressure waveforms}

Figure 2 shows the 2D-FDTD simulation and experimental results of the temporal pressure waveforms after passing through a hole with various diameters. The pressure waveforms without PC plate (in free space) were also shown in this figure due to comparison. In the experimental results, the time at the maximum discharge power was set to 0 seconds. The positive peak pressure increased with increasing hole

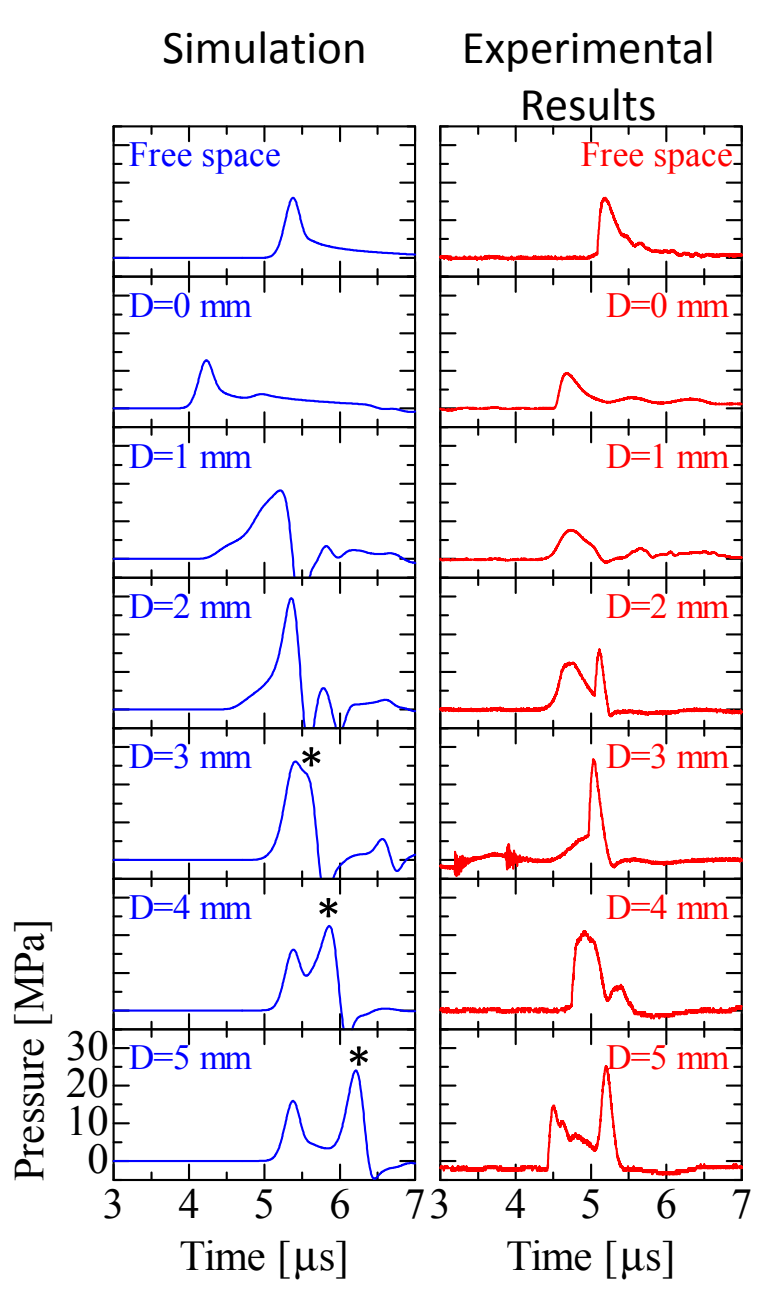

Fig. 2 2D-FDTD simulation and experimental results of pressure waveforms after passing through a hole with various diameters. The waveforms in free space also show due to comparison. Asterisk $(*)$ indicates the reflected pulse from the boundary between water and PC plate inside the hole. 


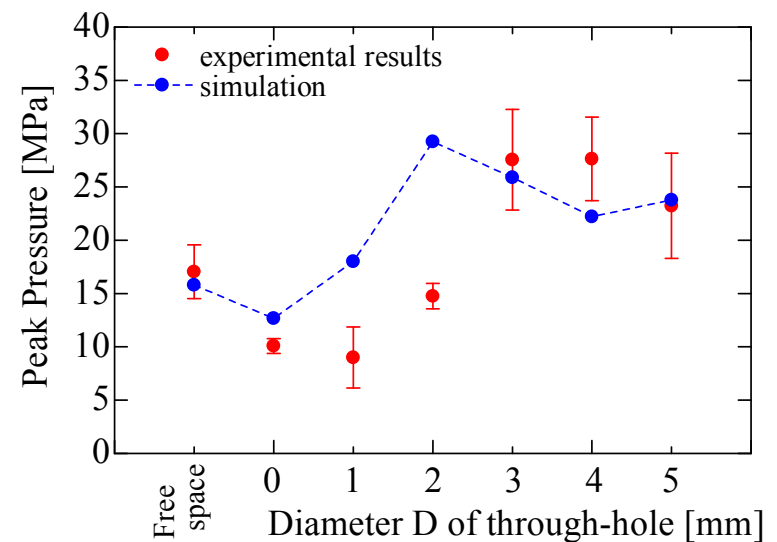

Fig. 3 Variations of the positive peak pressure, when the diameter of the through-hole was varied from 0 to $5 \mathrm{~mm}$. The results in free space were also shown due to comparison.

diameter. The negative pressure component was also observed after the positive pressure pulse, however, it was smaller than that of the simulation results. The reflected pulses from the boundary between water and PC plate of the hole inside were appeared following the first positive pulse, when the diameter was larger than $3 \mathrm{~mm}$ in this simulation. The increase in pressure is occurred due to the overlap between the direct pulse and the reflected pulses propagating in the hole inside.

3.2. Pressure variable after propagating through-hole

Figure 3 shows the variation of the positive peak pressure, when the diameter of the through-hole was varied from 0 to $5 \mathrm{~mm}$. The results in free space were also shown in this figure. Comparing the simulation with the experimental results, the diameters at which the maximum peak pressure was obtained were different. The positive peak pressure after propagating through-hole became maximum at diameter of $2 \mathrm{~mm}$ in simulation, whereas, of $3 \mathrm{~mm}$ in experiment. This difference indicates that the pressure source of the experimental model is not necessarily located only on the central axis. We suppose the formation of a linear pressure source along the discharge path. However, tendency of the peak pressure variation was similar. In this experimental results, the maximum value of the positive peak pressure became about 1.5 times large $(32 \mathrm{MPa})$, as compared with that $(20 \mathrm{MPa})$ in free space.

3.3. Pressure distribution on PC plate with cylindrical through-hole

Figures 4(a) and 4(b) show the pressure distribution along normal and parallel directions to the groove, respectively. A through-hole with the diameter of $4 \mathrm{~mm}$ was used in this measurement. The positive pressure values were measured by moving the hydrophone sensor along the horizontal direction from the central axis of the through-hole. Although the largest pressure value was obtained on the central axis, it was approximately $20 \mathrm{MPa}$ in free space. On the other hand, the pressure values larger than $20 \mathrm{MPa}$ were obtained in the through-hole range from the center to about $1.0 \mathrm{~mm}$, and decreased sharply at the position of $2 \mathrm{~mm}$ from the center. It was displayed that a maximum intensity over $30 \mathrm{MPa}$ was obtained at the hole center in this experiment (Fig. 4).

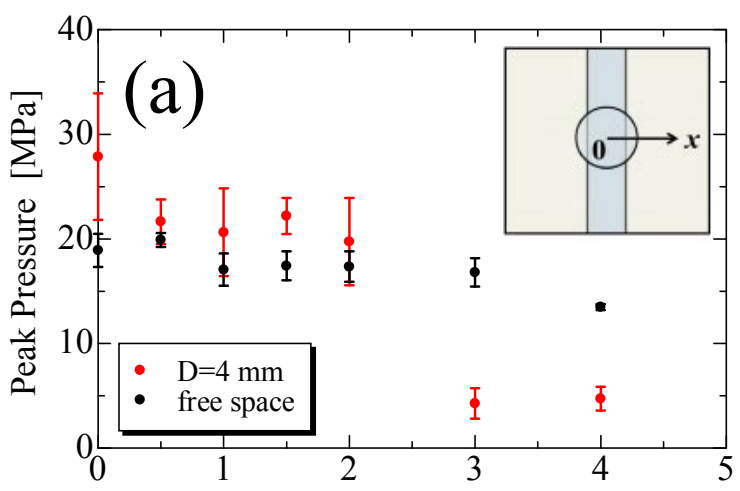

Position along $\mathrm{x}$-axis normal to a groove [mm]

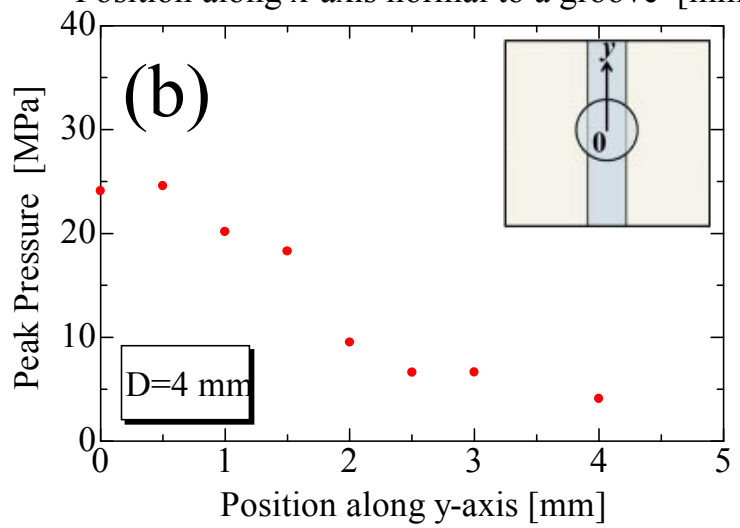

Fig. 4 Pressure distributions along (a) normal ( $x$-axis) and (b) parallel (y-axis) directions to the groove. A through-hole with the diameter of $4 \mathrm{~mm}$ was used in this measurement. The positive pressure values were measured by moving the hydrophone sensor along the horizontal direction from the central axis of the through-hole.

In transdermal drug delivery with a single photomechanical pressure, a peak pressure pulse exceeding $50 \mathrm{MPa}$ has been used and $35 \mathrm{MPa}$ is reported as the permeation pressure threshold for SC [4,10-13]. Proposed method is useful for reducing the applied energy used to generate pressure pulses and for obtaining high-intensity pressure pulses.

\section{Conclusion}

We have found an experimental condition which an intense pressure is formed by propagating single pulse through a cylindrical hole. According to our study, the ratio between the length and diameter of the through hole was 0.6 to 0.8 as suitable condition. When a pressure pulse was propagated through a $4 \mathrm{~mm}$ diameter cylindrical with the length of $5 \mathrm{~mm}$, a positive peak pressure value about 1.5 times larger than that in free space propagation was obtained on the central axis of the through-hole.

\section{Acknowledgments}

The authors thank Mr. Soma Saito and Mr. Takato Morita for technical assistance. The authors also thank Professor Naoki Osawa of Kanazawa Institute of Technology for lending us the equipment. This work was partly supported by JSPS KAKENHI Grant Number 18K04271. 


\section{References}

[1] C. H. Chaussy, W. Brendel and E. Schmiedt, "Extracorporeally induced destruction of kidney stones by shock waves," Lancet, 316, 1265-1268 (1980).

[2] C. D. Ohl and B. Wolfrum, "Detachment and sonoporation of adherent HeLa-cells by shock wave-induced cavitation," Biochim. Biophys. Acta Gen. Subj., 1624, 131-138 (2003).

[3] T. Hasebe, M. Kogi and K. Aizawa, "Effect of shock wave on the distribution of foreign substance introduced in human cells," IEICE Tech. Rep., US2016-21, pp. 11-16 (2016) (in Japanese).

[4] A. G. Doukas and N. Kollias, "Transdermal drug delivery with a pressure wave," Adv. Drug Deliv. Rev., 56, 559-579 (2004).

[5] T. Kobayashi and K. Aizawa, "Single underwater spark discharge induced shock wave propagated within the waveguide," Proc. 39th Symp. Ultrason. Electron., 39, 2P4-4 (2018).

[6] K. Yee, "Numerical solution of initial boundary value problems involving Maxwell's equations in isotropic media," IEEE Trans. Antennas Propag., 14, 302-307 (1966).

[7] S. Z. Wang, "Finite-difference time-domain approach to underwater acoustic scattering problems," J. Acoust. Soc. Am., 99, 1924-1931 (1996).
[8] F. Iijima, T. Tsuchiya and N. Endoh, "Analysis of characteristics of underwater sound propagation in the ocean by a finite difference time domain method," Jpn. J. Appl. Phys., 39, 3200-3204 (2000).

[9] R. L. Higdon, "Absorbing boundary conditions for difference approximations to the multidimensional wave equation," Math. Comput., 47, 437-459 (1986).

[10] S. Lee, D. J. McAuliffe, T. J. Flotte, N. Kollias and A. G. Doukas, "Photomechanical transcutaneous delivery of macromolecules," J. Invest. Dermatol., 111, 925-929 (1998).

[11] S. Lee, D. J. McAuliffe, N. Kollias, T. J. Flotte and A. G. Doukas, "Permeabilization and recovery of the stratum corneum in vivo: The synergy of photomechanical waves and sodium lauryl sulfate," Lasers Surg. Med., 29, 145-150 (2001).

[12] M. Ogura, S. Sato, M. Kuroki, H. Wakisaka, S. Kawauchi, M. Ishihara, M. Kikuchi, M. Yoshioka, H. Ashida and M. Obara, "Transdermal delivery of photosensitizer by the laser-induced stress wave in combination with skin heating," Jpn. J. Appl. Phys., 41, L814-L816 (2002).

[13] S. Lee, N. Kollias, D. J. McAuliffe, T. J. Flotte and A. G. Doukas, "Topical drug delivery in humans with a single photomechanical wave," Pharm. Res., 16, 1717-1721 (1999). 